Purdue University Purdue e-Pubs

\title{
Heart Failure in Hispanic Americans: Improving
} Cultural Awareness

\author{
Kenneth D. Melton \\ Karen J. Fell \\ Karen S. Yehle \\ Purdue University, kyehle@purdue.edu \\ Rosanne R. Griggs
}

Follow this and additional works at: http://docs.lib.purdue.edu/nursingpubs

Part of the Nursing Commons

\section{Recommended Citation}

Melton, Kenneth D.; Fell, Karen J.; Yehle, Karen S.; and Griggs, Rosanne R., "Heart Failure in Hispanic Americans: Improving Cultural Awareness" (2015). School of Nursing Faculty Publications. Paper 24.

http://dx.doi.org/10.1016/j.nurpra.2014.08.010 
Heart Failure in Hispanic Americans:

Improving Cultural Awareness

Kenneth D. Melton, MS, RN

Purdue University School of Nursing

College of Health and Human Sciences

502 N. University Street, JNSN 234

West Lafayette, IN 47907

E-mail: kmelton1@iuhealth.org

Office: 765-494-4023

Fax: 765-494-6339

Graduation Date: May 17, 2014

Karen J. Foli, PhD, RN (Corresponding Author)

Assistant Professor, Purdue University School of Nursing

College of Health and Human Sciences

502 N. University Street, JNSN 234

West Lafayette, IN 47907

E-mail: kfoli@purdue.edu

Office: 765-494-4023

Fax: 765-494-6339

Karen S. Yehle, PhD, FAHA

Associate Professor, Purdue University School of Nursing

College of Health and Human Sciences

502 N. University Street, JNSN 238

West Lafayette, IN 47907

E-mail: kyehle@purdue.edu

Office: $\underline{765-494-4011}$

Fax: 765-494-6339

Rosanne R. Griggs, PhD, FNP-BC

Clinical Associate Professor, Purdue University School of Nursing

College of Health and Human Sciences

502 N. University Street, JNSN 244

West Lafayette, IN 47907

E-mail: rgriggs@purdue.edu

Office: 765-494-4021

Fax: 765-494-6339 


\author{
Heart Failure in Hispanic Americans: \\ Improving Cultural Awareness
}

\begin{abstract}
Hispanic Americans are the largest and fastest growing ethnic group in the United States. Hispanic Americans have high rates of heart failure (HF) risk factors such as hypertension, diabetes mellitus, obesity, obstructive sleep disorders and dyslipidemia. Certain unique HF risk factors are prominent among Hispanic Americans that are uncommon in the general population, such as younger onset of valvular disease and Chagas disease. Advanced practice nurses providing care to Hispanic Americans have an ethical obligation to provide culturally competent care and assist these patients in overcoming barriers to healthcare so that they can effectively manage their HF.
\end{abstract}

KEY WORDS: Hispanic American, heart failure, risk factors, disease management, healthcare barriers 


\section{Heart Failure in Hispanic Americans: Improving Cultural Awareness}

Hispanic Americans (Hispanics) are a heterogeneous people from the Caribbean, Central and South American linked together by a shared Spanish culture. ${ }^{1}$ One of the fastest growing ethnicities in the United States (US), Hispanics account for more than half of the US population growth between 2000 and 2010. ${ }^{2}$ Rapid population growth will result in Hispanics representing one-third of the US population by $2050 .^{3}$

Heart failure (HF) is a chronic condition and major public health burden with prevalence rates and costs projected to increase in the US. ${ }^{4}$ Coronary artery disease causes the majority of HF cases in the US. ${ }^{5}$ While coronary artery disease is less common among Hispanics than nonHispanic Caucasian or African Americans, ${ }^{6}$ Hispanics often have high rates of common and unique causes of non-ischemic HF. Literature on HF among Hispanics is limited due to underrepresentation of Hispanics in HF studies or researchers enrolling only Hispanics of one national origin that may not be representative of this heterogeneous population.

As the Hispanic population continues to grow, advanced practice nurses (APNs) may treat more Hispanic patients with HF in clinical practice. Often these patients lack insurance, ${ }^{7}$ forgo care due to cost, ${ }^{8}$ have limited English proficiency and low health literacy ${ }^{9}$ which may influence health and healthcare access. These issues can challenge providers treating HA patients as providers attempt to promote healthy behaviors, provide health education, and diagnose, treat and manage HF symptoms. The purpose of this article is to summarize common and unique risk factors of HF in Hispanics, discuss barriers Hispanics endure in obtaining care, and identify interventions to improve the cultural competency of APNs.

\section{Epidemiology}


Heart failure prevalence rates differ significantly between Hispanics of different nationalities. The American Heart Association (AHA) estimates the prevalence of HF among Mexican Americans to be $1.9 \%$ for men and $1.1 \%$ for women, lower than both Caucasian and African Americans. ${ }^{6}$ A multiethnic cohort study on heart disease found that Hispanics have the second highest risk of developing HF after African Americans. ${ }^{6}$

Adult Hispanics have high prevalence rates of HF risk factors including overweight/obesity, diabetes mellitus, metabolic syndrome, physical inactivity, dyslipidemia, and uncontrolled hypertension. ${ }^{6}$ Hispanics with HF are more likely to be younger, uninsured, and have higher prevalence rates of diabetes mellitus and hypertension than Caucasian Americans. ${ }^{10}$ Additionally, elderly Hispanics with HF are more likely than elderly Caucasian Americans with HF to be readmitted to hospitals. ${ }^{11}$ Hispanics are more likely to have worse left ventricular hypertrophy ${ }^{12}$ and ejection fraction indicating a possible poorer prognosis. ${ }^{10}$

\section{The Role of the APN in Treating Hispanic Patients with HF}

The APN has a critical role in primary care to prevent, teach, diagnose, treat and manage chronic diseases while offering culturally competent care. Prevention of HF in patients requires APNs to provide health education and medical treatments to reduce a patient's risk profile of developing HF. The APN should work in collaboration with other medical specialty providers; cardiologists, internists, geriatricians, nurses, pharmacists, social workers, psychologists, physical therapists and dieticians to diagnosis, treat and manage HF symptoms. While certain risk factors may be more common in Hispanics, APNs should individualize care and work to reduce each individual's HF risk factors.

\section{Risk Factors}


Smoking. Smoking is a significant risk factor for HF. ${ }^{4}$ Providers must encourage Hispanic smokers to quit and explain the risks of smoking. Robles, Singh-Franco, and Ghin ${ }^{13}$ found that healthcare providers were more likely to discuss smoking cessation or offer nicotine replacement therapy to Caucasian patients than Hispanic patients. While Hispanic smokers may recognize the benefits of smoking cessation, providers may encounter resistance in accepting pharmaceutical aides to quit. A study of Hispanic American smokers found that the majority of participants rejected the use of medications for smoking cessation, especially Bupropion (Wellbutrin), due to its use to treat depression and the commonly held belief that an individual's ability to stop smoking was based on willpower and not medication aids. ${ }^{14}$

While Hispanic smokers may be resistant to smoking cessation interventions a metaanalysis revealed that smoking cessation interventions such as nicotine replacement therapy and counseling were highly effective in helping Hispanic patients quit smoking. ${ }^{15}$ Due to the effectiveness of pharmacologic and counseling treatment for smoking cessation, APNs should address and discuss cultural beliefs and socially contextual experiences, such as the stigma related to mental illnesses. In these ways, the APN may help Hispanic patients overcome beliefs pertaining to "willpower" and become more accepting of treatments.

Obesity, Diet and Physical Inactivity. Obesity is a recognized cardiovascular risk factor and increases an individual's risk of developing HF. ${ }^{4}$ Visceral abdominal obesity is a contributing factor to many diseases associated with HF, including type 2 diabetes, coronary arterial disease, myocardial infarction, dyslipidemia, hypertension, sleep apnea and metabolic syndrome. ${ }^{16}$ Mexican Americans are more likely to be obese or overweight compared to Caucasian Americans. ${ }^{6}$ 
Eating a heart healthy diet following the recommendations of the Dietary Approaches to Stop Hypertension is a component of ideal cardiovascular health. ${ }^{17}$ Mexican Americans are just as unlikely as Caucasian and African Americans to achieve at least four of the five AHA recommended key components of a healthy diet. ${ }^{6}$ The National Health and Nutritional Examination Survey (NHANES) found that Mexican American men consume more sweetened beverages than Caucasian or African American men and that Mexican Americans consume more carbohydrates than Caucasian or African Americans. ${ }^{6}$ A higher intake of sweeten beverages and carbohydrates has been shown to increase visceral obesity and the risk of coronary heart disease and diabetes. ${ }^{6}$

The US federal government and the AHA recommend 150 minutes of moderate physical activity for adults each week. ${ }^{17}$ A sedentary lifestyle increases one's risk of becoming overweight or obese. Hispanics are less likely to meet the physical activity recommended guidelines than other racial/ethnic groups. ${ }^{6}$ Social isolation may be a contributing factor to the high prevalence of physical inactivity among Hispanics. ${ }^{18}$

Hypertension. Hypertension is a known risk factor for $\mathrm{HF}^{4}$ The prevalence of hypertension is equal between Mexican and Caucasian Americans, however Mexican Americans are more likely to have poor blood pressure control, less likely to be aware of high blood pressure or be on an anti-hypertensive medication than Caucasian Americans. ${ }^{6}$ Middle and older adult Hispanics were more likely to have hypertension compared to Caucasian Americans. ${ }^{19}$ US born Cubans, Dominicans and Central/South Americans were less likely to have hypertension compared to other Hispanics, while foreign born Cubans, Puerto Ricans and Dominicans were more likely to have hypertension compared to other Hispanics. ${ }^{20}$ 
Outcome data from randomized controlled trials indicate that Hispanics receiving antihypertensive treatment were able to obtain better blood pressure control compared to Caucasian or African Americans participants if they had access to healthcare and medications. ${ }^{19}$ Hispanics on combination anti-hypertensive therapies were more likely to have a greater reduction in blood pressure compared to those on monotherapies. ${ }^{19}$ Barriers for Hispanics to obtain blood pressure control include lack of access to care and medications, dietary patterns, acculturation, and communication barriers. ${ }^{21}$

Diabetes Mellitus. Diabetes mellitus and insulin resistance have been linked as causative factors in the development of HF and worsens the progression of HF. ${ }^{22}$ The effect of diabetes on the heart is multifactorial resulting in both structural and functional changes. ${ }^{23}$ After adjusting for age, Hispanics over the age of 20 are more likely to have diabetes than any other racial/ethnic group except African Americans. ${ }^{24}$ It is projected that Hispanics will have the greatest increase in diabetes prevalence rate by 2050 compared to other racial/ethnic groups. ${ }^{6}$ After five years of follow-up, Nettleton et al. ${ }^{25}$ identified Hispanics as having the highest incidence rate of diabetes compared to Caucasian and African Americans. Mexican Americans and Puerto Ricans are more likely to have diabetes than Cuban Americans and Hispanics from Central and South America. ${ }^{24}$ Mexican Americans have the second highest prevalence rate of physician diagnosed diabetes after African Americans, but Mexican Americans have the highest prevalence of undiagnosed diabetes. ${ }^{6}$ Hispanics are less likely to self-monitor blood glucose and have worse glycemic control than Caucasian Americans. ${ }^{26}$

Dyslipidemia. Dyslipidemia is an indirect risk factor for HF as it is an independent risk factor for coronary artery disease and increased ratio of total cholesterol to high density lipoprotein levels increases HF risk. ${ }^{16}$ Mexican American men have worse total cholesterol, low 
density lipoprotein, triglycerides, and high density lipoprotein levels compared to males of other racial/ethnic groups, while Mexican American women have worse high density lipoprotein levels compared to Caucasian and African American women. ${ }^{6}$

Other HF Etiologies. Certain HF etiologies are common in Hispanics that may not be common in other US populations. Valvular heart disease is a known risk factor for HF, which may be caused by rheumatic fever. The incidence of rheumatic fever and rheumatic valvular disease has significantly decreased in the US, but continues to be endemic in other areas of the world including Central and South America. ${ }^{6}$ Hispanics who recently immigrated to the US or visited areas of world where rheumatic fever is endemic are at risk of developing HF. ${ }^{27}$ Hispanic patients who required mitral valve replacement were more likely to be younger, have a history of rheumatic fever and be diagnosed with $\mathrm{HF}^{28}$

Chagas disease is a zoonotic disease caused by the Trypanosoma cruzi, a parasite endemic to South and Central America, Mexico and south US, and is a major HF risk factor outside the US. ${ }^{29}$ Foreign-born immigrants arriving to the US from endemic areas may have asymptomatic infections that place the individual at risk of HF. Chagas induced cardiomyopathy and HF has a worse prognosis than non-Chagas cardiomyopathy and HF related to rapid heart remodeling and changes in electrical conduction. ${ }^{29}$ Clinical presentation of Chagas induced HF symptoms are based upon the extent of the cardiac damage and vary between individuals. Due to the infrequency of Chagas infections in the US, Chagas-related HF may be confused with idiopathic cardiomyopathy and providers should consider this as a cause of HF in foreign born patients or those who recently visited an endemic area.

Obstructive sleep disorders have been linked with $\mathrm{HF} ;{ }^{16}$ patients with obstructive sleep apnea and HF have a greater mortality rate than those HF patients that do not have obstructive 
sleep apnea. ${ }^{30}$ There is no published literature on the prevalence of obstructive sleep apnea in Hispanics; however research indicates that Hispanics are more likely to snore and have more frequent oxyhemoglobin desaturations per hour than other racial/ethnic groups. ${ }^{31}$

\section{Overcoming Healthcare Barriers for Hispanic Patients}

The diagnosis, treatment and management guidelines for HF are the same for Hispanics and other racial/ethnic groups. Therefore, APNs should be familiar with the current recommended HF guidelines. Treatment and care management plans however may need to be adjusted based upon the cultural preferences of the patient. Healthcare disparities continue to be a major barrier for Hispanic patients. To reduce healthcare disparities, providers should be familiar with these barriers (Table 1) and appropriate interventions that can assistant in providing culturally competent care that can eliminate many disparities for Hispanic patients (Table 2).

\section{Lack of Quality Healthcare}

Poor quality of care is a significant healthcare disparity among Hispanic patients with HF. Hispanic patients are likely to state that healthcare providers do not involve them in decision making or spend enough time explaining or answering their healthcare concerns. ${ }^{32}$ Early studies of Hispanic HF patients indicated that Hispanics were less likely than Caucasian and African Americans to receive an assessment of left ventricular ejection fraction, ${ }^{33}$ be discharged on an ACE inhibitor ${ }^{33}$ or receive complete discharge instructions at the time of hospital discharge. ${ }^{34}$ While the overall delivery of healthcare to Hispanic HF patients at hospitals has improved ${ }^{35}$ an examination of Medicare Hospital Quality measures indicate that Hispanic patients with HF are less likely to receive smoking cessation counseling and discharge instruction especially at poorer performing hospitals. ${ }^{36}$ Hispanic HF patients are less likely to 
receive follow-up consultations with a cardiologist than Caucasian Americans patients, ${ }^{37}$ have a usual source of healthcare ${ }^{38}$ or participate in clinical trials ${ }^{27}$ than other racial/ethnic groups.

\section{Lack of Health Insurance}

Two of the three top barriers to accessing healthcare among Hispanics are the lack of health insurance and the high cost of healthcare. ${ }^{8}$ In 2012, 29.2\% of Hispanics lacked health insurance, the highest percentage among racial/ethnic groups. ${ }^{7}$ Lack of insurance is more common among foreign born Hispanics, especially among undocumented individuals that are ineligible for government assistance programs such as Medicaid or the subsidies and insurance exchanges provided by the Affordable Care Act. ${ }^{39}$ Poverty and employment in low-income occupations that do not offer insurance prevent many Hispanics from obtaining employer based insurance or purchasing individual health insurance policies. ${ }^{32}$

Regardless of insurance status many Hispanics report difficulty in paying for

healthcare. ${ }^{27}$ Lack of insurance results in Hispanic patients receiving fewer referrals to specialists, reduces access to preventive care, results in later detection of life-threatening conditions, and infrequent management of chronic diseases, all which worsen healthcare disparities. $^{38}$

\section{Communication}

Ineffective communication between Hispanics and their healthcare providers may result in poor care. One-third of Hispanics report limited English proficiency and 45\% report limited health literacy. ${ }^{9}$ Limited health literacy increases a patient's risk of a poor health outcome. ${ }^{40}$ Limited English proficiency and health literacy in Hispanics is associated with poor health reporting. ${ }^{9}$ Conversely, Hispanics who report a higher level of acculturation are more likely to have improved health literacy. ${ }^{38}$ 
Use of an interpreter is essential and recommended when healthcare providers communicate with non-English speaking Hispanics, and using a trained interpreter improves health outcomes in Hispanics. ${ }^{41}$ Using simple terminology and visual aids can assist providers in communicating. ${ }^{32}$ When communicating with patients, providers should not appear rushed. A personalized conversation can cultivate a relationship of trust with Hispanic patients. ${ }^{42}$ Interventions and education material should use culturally appropriate references in order to have success. $^{32}$

Respeto is a Hispanic cultural value in which respect and differential behavior is demonstrated to those in authority. ${ }^{32}$ This distribution of power favoring the provider in the patient-provider relationship may impair communication. Due to the respeto value, a patient may tell the provider that they will adhere to prescribed treatments when they have no intention of following the provider's instructions or the patient may not ask questions as they may perceive this as a sign of disrespect.

Providers should recognize the importance of nonverbal communication. To avoid miscommunication, providers should be familiar with cultural mannerisms and body language of Hispanics. Providers should avoid prolonged direct eye contact and sit close to Hispanic patients in order to show respect. ${ }^{43}$ The provider's mannerisms and behavior should be open and friendly as Hispanics view the provider-patient interaction as part of the healing process. ${ }^{32}$

The Hispanic culture is typically present-oriented and more flexible with time. ${ }^{32}$ They may be late to healthcare appointments. Due to the present-orientation, interventions that stress short term goals are more likely to be accepted..$^{32}$

\section{Cultural Competency among Providers}


Understanding the Hispanic culture will help APNs provide culturally competent care. Common cultural concepts that APNs should be familiar with include: family, alternative medications/therapies, and gender roles.

Family. Family or familismo is central to the Hispanic culture and may override the needs of the individual. Family is often included in the decision making process, especially in managing critical health-care decisions, such as those associated with HF. ${ }^{43}$ Hispanic patients will typically seek advice not only from within the nuclear family, but from extended family on decisions pertaining to medications, self-medicating, the use of traditional healers called curanderos, and folk remedies. ${ }^{32}$ This dependence on input from family members can result in patients delaying medical treatment. ${ }^{42}$ Family celebrations are commonly associated with overeating and unhealthy foods. This can be problematic for Hispanics with HF since interpersonal conflict may arise in refusing foods, a sign of disrespect in the Hispanic culture. ${ }^{42}$ Encouraging family member participation in treatment plans and lifestyle modifications may improve adherence. ${ }^{43}$

Alternative Medications and Therapies. Principles of current Hispanic alternative medications and therapies are rooted in the mixing of cultural beliefs from the native people of Central and South America. ${ }^{44}$ These beliefs have given rise to the traditional Hispanic medical therapy related to hot and cold diseases and treatments. Diseases that are considered hot are treated with cold remedies, and cold diseases are treated with hot remedies to restore balance in the body. 32

Often Hispanics will seek out curanderos for treatments include hot/cold remedies, herbs, rest, foods and massage. ${ }^{32}$ Curanderos are highly respected in the Hispanic culture. Unfortunately the use of curanderos may increase self-medication, delay seeking treatment from 
healthcare providers, and distrust of healthcare providers, reducing adherence to prescribed treatments. ${ }^{32,44}$ Up to $78 \%$ of Hispanics fail to disclose the use of curanderos and alternative treatments to providers because providers fail to ask. ${ }^{32}$

It should be standard practice for APNs to assess Hispanic patients about the use of herbal medications, hot/cold therapies, alternative medications and whether the patient visits a curandero for treatment. Having an active and informed discussion is essential in treating patients as a lack of respect for these alternative therapies may result in decreased regimen adherence or potential drug interactions. ${ }^{44}$ Providers should emphasize the benefits of prescribed regimens since some Hispanic patients may prefer alternative medications and therapies. ${ }^{32}$ Finally, APNs should incorporate a patient's alternative therapies, if not contraindicated, which may enhance the patient-provider relationship and improve adherence to prescribed treatments. ${ }^{44}$

Gender Roles. Traditional gender roles are common in the Hispanic culture. Machismo is a concept associated with masculinity that may result in men not seeking healthcare or refusing treatment associated with weakness. ${ }^{42}$ Due to specific gender roles, women often are the primary caregivers preparing food for the family and should therefore be encouraged to participate in the patient's HF education about dietary restrictions. ${ }^{42}$ Hispanic American male patients often are accompanied to provider visits by spouses or female family members. These female family members should be included in the patient's education, as they often encourage men to seek care and adhere to treatment. ${ }^{42}$

\section{Conclusion}

As the fastest growing population group, Hispanic American's healthcare concerns will be an important issue for the US healthcare system. Hispanics have an increased risk of cardiovascular diseases, including HF. Advanced practice nurses will be essential in bringing 
culturally competent, informed HF treatment plans and educational strategies to Hispanics. The APNs understanding of Hispanic cultural components including familismo, machismo, and alternative medications/therapies will help ensure the patient receives high quality healthcare, and promote effective communication with Hispanic patients. 


\section{References}

1. Humes KR, Jones NA, Ramirez RR. 2010 Census Briefs C2010BR-02: Overview of Race and Hispanic Origin: 2010. Washington, DC: US Census Bureau; 2011. http://www.census.gov/prod/cen2010/briefs/c2010br-02.pdf. Accessed March 25, 2014.

2. Ennis SR, Rios-Vargas M, Albert NG. 2010 Census Briefs C2010BR-04: The Hispanic Population: 2010. Washington, DC: US Census Bureau; 2011.

3. Ortman JM, Guarneri CE. United States Population Projections: 2000 to 2050. Washington, DC: US Census Bureau; 2009. http://www.census.gov/population/projections/files/analytical-document09.pdf. Accessed March 25, 2014.

4. Heidenreich PA, Albert NM, Allen LA, et al. Forecasting the impact of heart failure in the United States: a policy statement from the American Heart Association. Circulation. Heart failure. 2013;6(3):606-619.

5. Hunt SA, Abraham WT, Chin MH, et al. 2009 focused update incorporated into the ACC/AHA 2005 Guidelines for the Diagnosis and Management of Heart Failure in Adults: a report of the American College of Cardiology Foundation/American Heart Association Task Force on Practice Guidelines: developed in collaboration with the International Society for Heart and Lung Transplantation. Circulation. 2009;119(14):e391-479.

6. Go AS, Mozaffarian D, Roger VL, et al. Heart disease and stroke statistics-2014 update: a report from the American Heart Association. Circulation. 2014;129(3):e28-292.

7. DeNavas-Walt C, Proctor BD, Smith JC. Current Population Reports P60-243: Income, Poverty, and Health Insurance Coverage in the United States: 2012. Washington, DC:

US Census Bureau; 2012. http://www.census.gov/prod/2012pubs/p60-243.pdf. Accessed March 25, 2014.

8. Long JM, Sowell R, Bairan A, Holtz C, Curtis AB, Fogarty KJ. Exploration of commonalities and variations in health related beliefs across four Latino subgroups using focus group methodology: implications in care for Lations with Type 2 Diabetes. J.Cult Divers. 2012;19(4):133-142.

9. Sentell T, Braun KL. Low health literacy, limited English proficiency, and health status in Asians, Latinos, and other racial/ethnic groups in California. J Health Commun. 2012;17(Suppl 3):82-99.

10. Thomas KL, Hernandez AF, Dai D, et al. Association of race/ethnicity with clinical risk factors, quality of care, and acute outcomes in patients hospitalized with heart failure. Am Heart J. 2011;161(4):746-754.

11. Rodriguez F, Joynt KE, Lopez L, Saldana F, Jha AK. Readmission rates for Hispanic Medicare beneficiaries with heart failure and acute myocardial infarction. Am Heart J. 2011;162(2):254-261.e253.

12. Rodriguez CJ, Diez-Roux AV, Moran A, et al. Left ventricular mass and ventricular remodeling among Hispanic subgroups compared with non-Hispanic blacks and whites: MESA (Multi-ethnic Study of Atherosclerosis). J Am Coll Cardiol. 2010;55(3):234-242.

13. Robles GI, Singh-Franco D, Ghin HL. A review of the efficacy of smoking-cessation pharmacotherapies in nonwhite populations. Clin Ther. 2008;30(5):800-812.

14. Levinson AH, Borrayo EA, Espinoza P, Flores ET, Perez-Stable EJ. An exploration of Latino smokers and the use of pharmaceutical aids. Am J Prev Med. 2006;31(2):167-171. 
15. Webb MS, Rodriguez-Esquivel D, Baker EA. Smoking cessation interventions among Hispanics in the United States: a systematic review and mini meta-analysis. Am J Health Promot. 2010;25(2):109-118.

16. Schocken DD, Benjamin EJ, Fonarow GC, et al. Prevention of heart failure: A scientific statement from the American Heart Association Councils on Epidemiology and Prevention, Clinical Cardiology, Cardiovascular Nursing, and High Blood Pressure Research; Quality of Care and Outcomes Research Interdisciplinary Working Group; and Functional Genomics and Translational Biology Interdisciplinary Working Group. Circulation. 2008;117(19):2544-2565..

17. Lloyd-Jones DM, Hong Y, Labarthe D, et al. Defining and setting national goals for cardiovascular health promotion and disease reduction: The American Heart Association's strategic impact goal through 2020 and beyond. Circulation. 2010;121(4):586-613.

18. Willey JZ, Paik MC, Sacco R, Elkind MS, Boden-Albala B. Social determinants of physical inactivity in the Northern Manhattan Study (NOMAS). J Community Health. 2010;35(6):602-608.

19. Guzman NJ. Epidemiology and management of hypertension in the Hispanic population: a review of the available literature. Am J Cardiovasc Drugs. 2012;12(3):165-178.

20. Pabon-Nau LP, Cohen A, Meigs JB, Grant RW. Hypertension and diabetes prevalence among U.S. Hispanics by country of origin: The National Health Interview Survey 20002005. J Gen Intern Med. 2010;25(8):847-852.

21. Perez A. Self-management of hypertension in Hispanic adults. Clin Nurs Res. 2011;20(4):347-365.

22. Bui AL, Horwich TB, Fonarow GC. Epidemiology and risk profile of heart failure. Nat Rev Cardiol. 2011;8(1):30-41.

23. Murarka S, Movahed MR. Diabetic cardiomyopathy. J Card Fail. 2010;16(12):971-979.

24. Centers of Disease Control and Prevention. National Diabetes Fact Sheet: National Estimates and General Information on Diabetes and Prediabetes in the United States, 2011. Altanta, GA: US Department of Health and Human Services, Centers for Disease Control and Prevention; 2011. http://www.cdc.gov/diabetes/pubs/pdf/ndfs_2011.pdf. Accessed March 25, 2014.

25. Nettleton JA, Steffen LM, Ni H, Liu K, Jacobs DR, Jr. Dietary patterns and risk of incident type 2 diabetes in the Multi-Ethnic Study of Atherosclerosis (MESA). Diabetes Care. 2008;31(9):1777-1782.

26. Campbell JA, Walker RJ, Smalls BL, Egede LE. Glucose control in diabetes: The impact of racial differences on monitoring and outcomes. Endocrine. 2012;42(3):471-482.

27. Vivo RP, Krim SR, Cevik C, Witteles RM. Heart failure in Hispanics. J Am Coll Cardiol. 2009;53(14):1167-1175.

28. DiGiorgi PL, Baumann FG, O'Leary AM, et al. Differences in mitral valve disease presentation and surgical treatment outcome between Hispanic and non-Hispanic patients. Ethn Dis. 2008;18(3):306-310.

29. Nunes MC, Dones W, Morillo CA, Encina JJ, Ribeiro AL. Chagas disease: an overview of clinical and epidemiological aspects. J Am Coll Cardiol. 2013;62(9):767-776.

30. Mannarino MR, Di Filippo F, Pirro M. Obstructive sleep apnea syndrome. Eur J Intern Med. 2012;23(7):586-593. 
31. Loredo JS, Soler X, Bardwell W, Ancoli-Israel S, Dimsdale JE, Palinkas LA. Sleep health in U.S. Hispanic population. Sleep. 2010;33(7):962-967.

32. Askim-Lovseth MK, Aldana A. Looking beyond "affordable" health care: Cultural understanding and sensitivity-necessities in addressing the health care disparities of the U.S. Hispanic population. Health Mark Q. 2010;27(4):354-387.

33. Correa-de-Araujo R, Stevens B, Moy E, Nilasena D, Chesley F, McDermott K. Gender differences across racial and ethnic groups in the quality of care for acute myocardial infarction and heart failure associated with comorbidities. Womens Health Issues. 2006;16(2):44-55.

34. Albert NM, Fonarow GC, Abraham WT, et al. Predictors of delivery of hospital-based heart failure patient education: a report from OPTIMIZE-HF. J Card Fail. 2007;13(3):189-198.

35. Vivo RP, Krim SR, Krim NR, et al. Care and outcomes of Hispanic patients admitted with heart failure with preserved or reduced ejection fraction: findings from get with the guidelines-heart failure. Cir Heart Fail. 2012;5(2):167-175.

36. Hasnain-Wynia R, Kang R, Landrum MB, Vogeli C, Baker DW, Weissman JS. Racial and ethnic disparities within and between hospitals for inpatient quality of care: an examination of patient-level Hospital Quality Alliance measures. J Health Care Poor Underserved. 2010;21(2):629-648.

37. Cook NL, Ayanian JZ, Orav EJ, Hicks LS. Differences in specialist consultations for cardiovascular disease by race, ethnicity, gender, insurance status, and site of primary care. Circulation. 2009;119(18):2463-2470.

38. Vega WA, Rodriguez MA, Gruskin E. Health disparities in the Latino population. Epidemiol Rev. 2009;31(1):99-112.

39. Kenney GM, Huntress M. The Affordable Care Act: Coverage Implications and Issues for Immigrant Families. Washington, DC: US Department of Health and Human Services; 2012. http://aspe.hhs.gov/hsp/11/ImmigrantAccess/Coverage/ib.shtml. Accessed March 25, 2014.

40. Berkman ND, Sheridan SL, Donahue KE, Halpern DJ, Crotty K. Low health literacy and health outcomes: an updated systematic review. Ann Intern Med. 2011;155(2):97-107.

41. Cersosimo E, Musi N. Improving treatment in Hispanic/Latino patients. Am J Med. 2011;124(10 Suppl):S16-21.

42. Caballero AE. Understanding the Hispanic/Latino patient. Am J Med. 2011;124(10 Suppl):S10-15.

43. Hicks D. Cultural competence and the Hispanic population. Medsurg Nurs. 2012;21(5):314-315.

44. Ortiz BI, Shields KM, Clauson KA, Clay PG. Complementary and alternative medicine use among Hispanics in the United States. Ann Pharmacother. 2007;41(6):994-1004. 
Table 1:

Common healthcare barriers for Hispanic American patients

Barriers

- Lack of quality healthcare

- Lack of health insurance

- Communication

$\circ \quad$ Limited English proficiency

- Limited health literacy

- Respeto

- Hispanic culture communication behavior

- Decreased awareness of Hispanic American culture among providers

- Family

- Alternative medications and therapies

- Gender roles 


\section{Table 2:}

Cultural Competent Interventions to Eliminate Healthcare Disparities among Hispanic Americans

- Cultural competency education for providers

- Monitor for quality outcomes and evaluate provider adherence to evidence-based practices with Hispanic patients

- Enhance comanagement of patient care between cardiologists and primary care providers when patient referred from a community health center

- Increase Hispanic participation in NIH funded HF research trials

- Improve access to health insurance

- Use of interpreter services

- Use of cultural appropriate and easy to read educational material

- Understanding of Hispanic cultural communication behaviors

○ Slow paced conversation

- Avoid direct eye contact

O Open and friendly nonverbal communication

- Present orientation time frame and using short term treatment goals

- Understanding of the Hispanic Respeto value system and how power distribution is imbalanced towards the provider

- Include family members, especially female family members in treatment discussion with consent of patient

- Familiarity, understanding and inclusion of Hispanic alternative therapies and the use of curanderos as appropriate 